Research Article

\title{
A Novel Stick-Slip Type Rotary Piezoelectric Actuator
}

\author{
Yuan Wang, Minglong Xu (D), Shubao Shao, Siyang Song, and Yan Shao \\ State Key Laboratory for Strength and Vibration of Mechanical Structures, School of Aerospace, Xi'an Jiaotong University, \\ Xi'an 710049, China \\ Correspondence should be addressed to Minglong Xu; mlxu@xjtu.edu.cn
}

Received 8 February 2020; Revised 18 April 2020; Accepted 30 April 2020; Published 27 May 2020

Academic Editor: Antonio Gloria

Copyright (c) 2020 Yuan Wang et al. This is an open access article distributed under the Creative Commons Attribution License, which permits unrestricted use, distribution, and reproduction in any medium, provided the original work is properly cited.

\begin{abstract}
A novel stick-slip rotary piezoelectric actuator is designed for optical use. The actuator is proposed, fabricated, and tested with the aim of realizing both fine resolution and a long stroke. The dynamic model of the actuator is established, and simulations are performed to discover how the input driving voltage affects the stick-slip motion of the actuator. An experimental system is built to evaluate the performance of the actuator at different frequencies, voltages, and numbers of driving piezoelectric stacks. Experimental results show that the minimal output stepping angle is $3.5 \mu \mathrm{rad}(0.2$ millidegrees) under a sawtooth waveform having a voltage of $13 \mathrm{~V}$ and frequency of $3000 \mathrm{~Hz}$ and that the velocity reaches $0.44 \mathrm{rad} / \mathrm{s}\left(25^{\circ} / \mathrm{s}\right)$ under a sawtooth waveform having a voltage of $93 \mathrm{~V}$ and frequency of $3000 \mathrm{~Hz}$, while the stroke is infinite. The proposed actuator provides stable and accurate rotary motion and realizes a high velocity.
\end{abstract}

\section{Introduction}

Piezoelectric actuators have been widely used in many fields [1], such as biomedical engineering [2], semiconductor manufacturing [3], optics focusing, and scanning microscopy [4], because of their compact size, large output force, rapid response, and high resolution [5, 6]. According to their working principles, piezoelectric actuators can be divided into direct-drive actuators, ultrasonic actuators, inchworm actuators, and inertial actuators. Direct-drive actuators have a high positioning resolution and compact structure but limited stroke [7-9]. Ultrasonic actuators have advantages for high-speed use but generate much heat and have wear problems [10-13]. Inchworm actuators produce a large output force but their structure and control system are complicated [14-17]. Inertial actuators have a compact structure, long stroke, and high resolution and are more promising for low-load precision applications [18-20]. There are two types of inertial actuator: the impact-inertial type and stick-slip type. This paper mainly discusses the latter.

Inertial stick-slip actuators [21-24] can be categorized by their control approach as signal-control type $[5,25]$, friction-control type [26], and mechanism-control type [27, 28] actuators. A signal-control actuator uses an excitation signal having an asymmetric waveform to generate unequal speed in two directions. The mechanism-control actuator uses an asymmetric mechanical structure to realize asymmetric operating motion. The friction-control actuator achieves motion by changing the friction force on the surface between the moving part and base in different motion stages.

Considering that the structure of the mechanism-control actuator is usually too complex and bulky for application and the friction-control actuator has drawbacks of low precision and limited stability [18], we propose a novel stickslip piezoelectric rotary actuator based on the signal-control method. The proposed rotary piezoelectric actuator is designed for an optical path adjustment system. The actuator provides precision angle displacement of at least $5 \mu \mathrm{rad}$, with a high velocity, infinite stroke, and structure for light to pass through, while having a relatively small volume and low weight.

The remainder of the paper is organized as follows. Section 2 clarifies the configuration and working principle of the actuator. Section 3 analyzes the dynamic model and discusses simulation results. Section 4 describes the experimental system and results for the actuator's characteristics. Section 5 presents conclusions. 


\section{Configuration and Operating Principle}

2.1. Configuration. Figure 1 depicts the configuration of the proposed rotary piezoelectric actuator. The actuator mainly comprises six components: a base, a bearing, two piezoelectric stacks placed in a rhombic mechanism, two preload springs, a joint plate, and a shell.

The bearing's inner ring is interference fitted on the base while the bearing's outer ring rotates freely and smoothly. Each piezoelectric stack is nested in a rhombic mechanism, which is used to protect the fragile piezoelectric stack and properly control the output displacement of the piezoelectric stack. The rhombic mechanism contains a friction block that is in contact with the bearing's outer ring and a fixing hole that is used to fix the mechanism on the surface of the base. The preload springs produce sufficient pressure between the friction block and outer ring of the bearing. The joint plate is interference fitted on the bearing's outer ring so that it rotates as the bearing rotates. The shell, which prevents the actuator from the environment, is fixed on the joint plate by screws and also rotates with the bearing.

The dimensions of the actuator are $56 \mathrm{~mm} \times$ $56 \mathrm{~mm} \times 12 \mathrm{~mm}$. The diameter of the aperture is $24 \mathrm{~mm}$. The dimensions of the bearing are $25 \mathrm{~mm} \times 37 \mathrm{~mm} \times 7 \mathrm{~mm}$. The dimensions of each piezoelectric stack are $3 \mathrm{~mm} \times$ $3 \mathrm{~mm} \times 9 \mathrm{~mm}$. The base, joint plate, shell, and rhombic mechanism of the piezoelectric stack are all made of Al7075 aluminum alloy.

The structure of the rotary actuator is designed for an optical path adjustment system as discussed in Section 1. The structure has an aperture at the center to let light pass through. The actuator rotates step by step to realize an unlimited stroke through stick-slip motion. Owing to the rhombic mechanism, the displacements of the piezoelectric stacks can be designed to have precision resolution. Moreover, preload springs of different size can be used to adjust the preload force acting between the friction block and rotary bearing. The proposed actuator has a compact structure and is lightweight.

2.2. Operating Principle. The operating principle and force analysis of the proposed piezoelectric rotary actuator are illustrated in Figure 2. When the piezoelectric stack is driven by a sawtooth waveform, it undergoes extension and contraction deformation, resulting in the friction block moving with respect to the bearing in a stick-slip manner.

(1) Initial state: when time $t=t_{0}$, no voltage is applied, the piezoelectric stack is not deformed, and there is no movement between the friction block and bearing.

(2) Slow extension stage: from times $t_{0}$ to $t_{1}$, the applied voltage increases slowly, the piezoelectric stack extends slowly, and the friction block sticks on the bearing, rotating the bearing through a small angle $\theta$.

(3) Quick contraction stage: from times $t_{1}$ to $t_{2}$, the applied voltage decreases rapidly and the piezoelectric stack contracts rapidly. At the same time, the rotor remains still while the friction block returns to its original position; that is, the friction block slips on the bearing.

Afterward, the rotor rotates though a small angle $\theta$ anticlockwise, and the friction block and piezoelectric stack return to the same position as for the initial state. Through the repeating cycle of the sawtooth wave voltage, the actuator outputs an infinite stroke step by step. The actuator rotates clockwise if we change the applied voltage such that it first increases rapidly and then decreases slowly.

2.3. Design of the Rhombic Mechanism. The rhombic mechanism is commonly used to amplify the deformation of the piezoelectric stack. This article uses the same principle to design the rhombic mechanism. The purpose is to constrain the displacement of the piezoelectric stack and protect the piezoelectric stack. The designed mechanism is actually arcshaped mechanism. The groove is processed by slow wire cutting inside the mechanical structure, and the piezoelectric stack is placed in the groove for driving. The length of the groove is slightly shorter than the original length of the piezoelectric stack by $5-10 \mu \mathrm{m}$, so the piezoelectric stack can be preloaded.

In the finite element analysis we can simplify the piezoelectric stack as a homogeneous solid material which will produces equivalent deformation with respect to voltage input [29]. The structure of the designed mechanism and finite element analysis results are shown in Figure 3. The deformation which is tested by experiment method of the piezoelectric stack driven by the voltage of $120 \mathrm{~V}$ is $9 \mu \mathrm{m}$. The simulation results of the deformation and stress diagram of the driving part constrained by the rhombic mechanism at the voltage of $120 \mathrm{~V}$ are shown in Figure 3. The maximum displacement is $7.1 \mu \mathrm{m}$, which is a loss of $1.9 \mu \mathrm{m}$ compared to the unconstrained displacement. The maximum stress is 113.9 $\mathrm{MPa}$, which is less than the yield stress of $500 \mathrm{MPa}$ for aluminum alloy 7075 . The first natural frequency of the mechanism is $2734 \mathrm{~Hz}$, and the second natural frequency is $6254 \mathrm{~Hz}$.

\section{Dynamic Model and Simulation}

3.1. Dynamic Model. In the dynamic model depicted in Figure 4, we simplify the rhombic mechanism and piezoelectric stack as a spring-damping-mass system, referred to as the PE subsystem. The spring stiffness and damping coefficient are denoted as $k$ and $c$, respectively. When the driving voltage is applied to the piezoelectric stack, a driving force $F_{p}$ is generated, and a friction force $F_{f}$ is generated on the contacting surface of the PE part and rotor part. $F_{f}$ drives the rotor with stick-slip motion.

This dynamic model is governed by the following equations:

$$
\begin{aligned}
m \ddot{x}+c \dot{x}+k x & =F_{p}-F_{f}, \\
J \ddot{\theta} & =M_{f}-M_{\text {load }},
\end{aligned}
$$




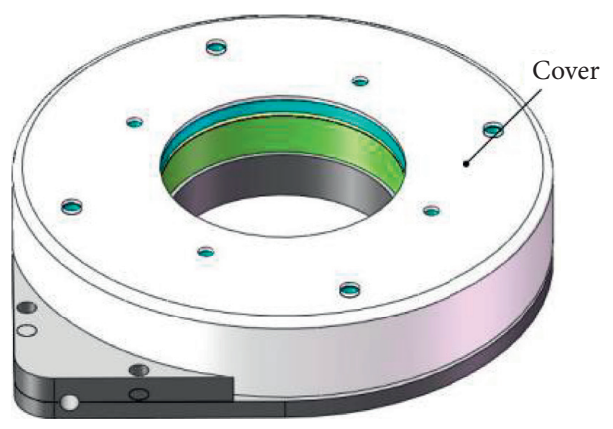

(a)

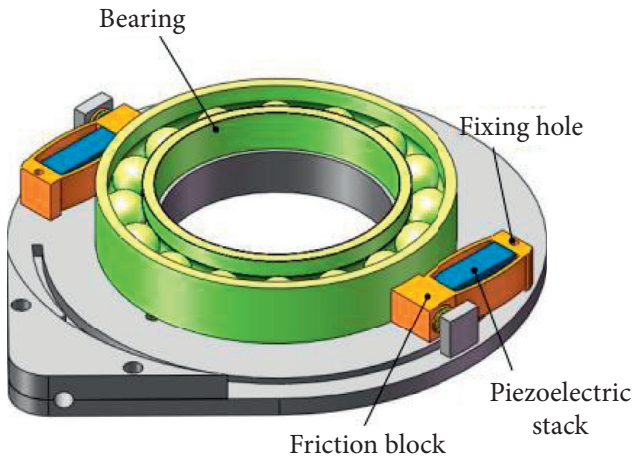

(b)

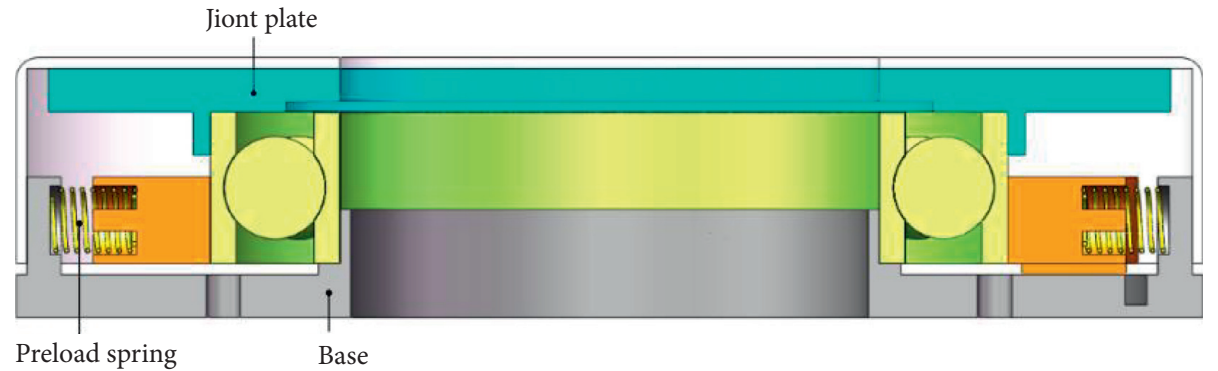

(c)

Figure 1: Configuration of the proposed piezoelectric rotary actuator. (a) 3D model. (b) Inside view. (c) A section.

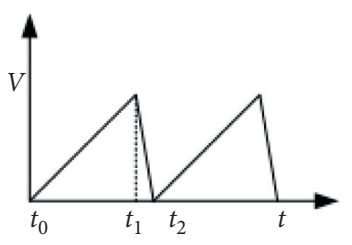

(a)

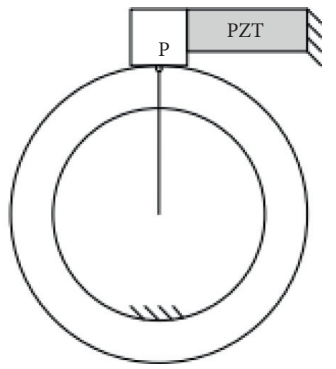

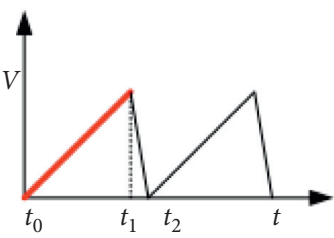

(b)
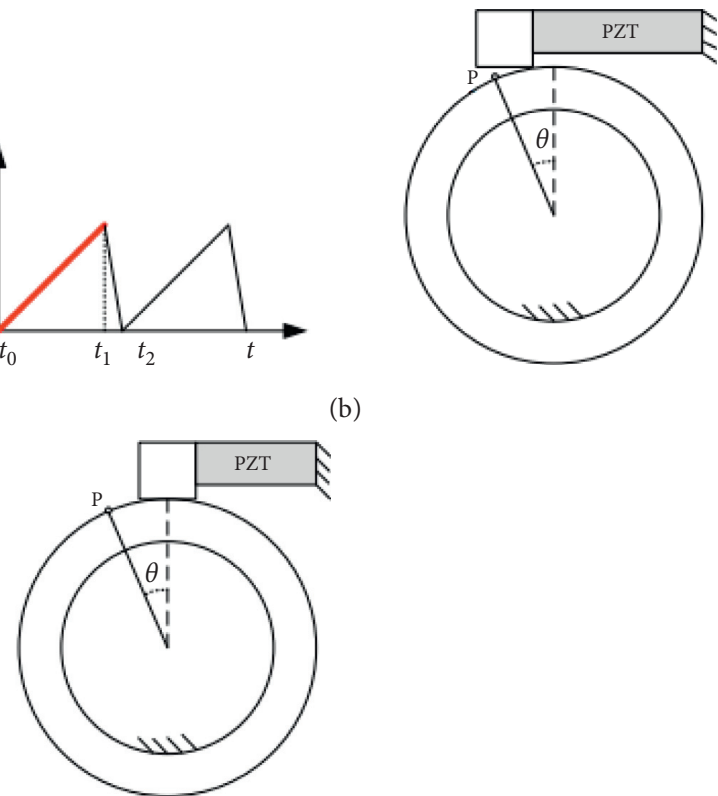

(c)

Figure 2: The operating principle.

where $F_{p}=k_{p} \cdot d_{e} \cdot V_{(t)}$ is determined by the properties of the PE subsystem, $J$ is the rotational inertia, $M_{f}$ is the output torque, $M_{\text {load }}$ is the load torque, $\ddot{\theta}$ is the angular acceleration, and $F_{f}$ is the friction force acting between the rotor and PE subsystem.
Figure 5 shows the deflected bristle in LuGre friction model. The friction force acting between the rotor and PE subsystem is dominated by presliding and is properly described by the LuGre friction model [30]: where $\sigma_{0}$ is the contact stiffness; $\sigma_{1}$ is the damping of the tangential 


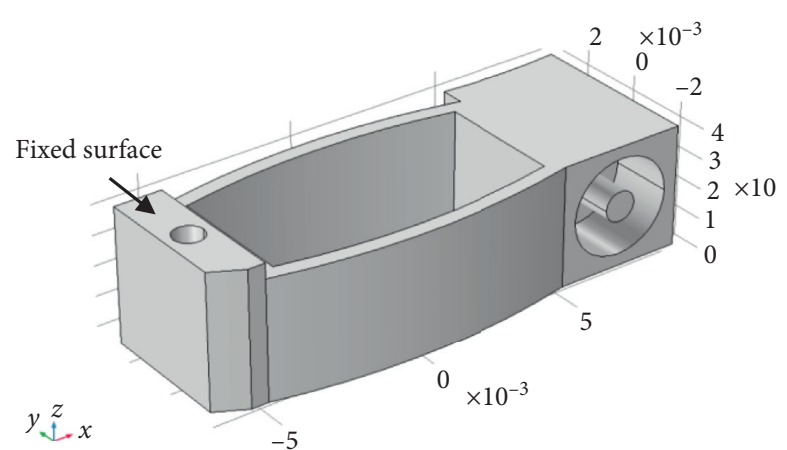

(a)

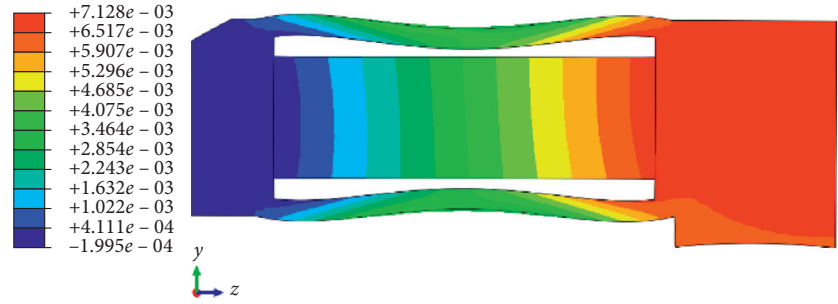

(b)

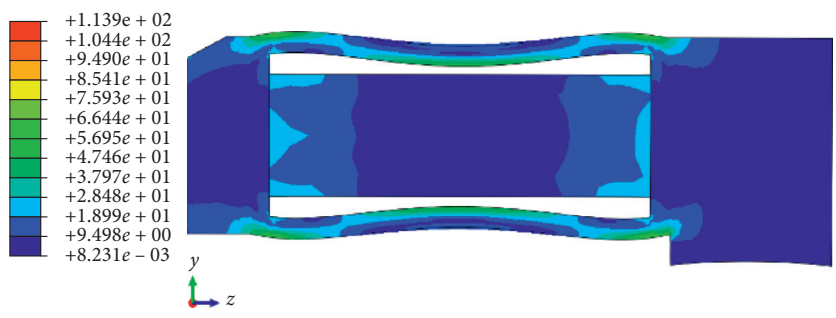

(c)

Figure 3: The design and analyze of rhombic mechanism. (a) Model sketch. (b) Deformation under driving voltage of 120 V. (c) Stress under driving voltage of $120 \mathrm{~V}$.

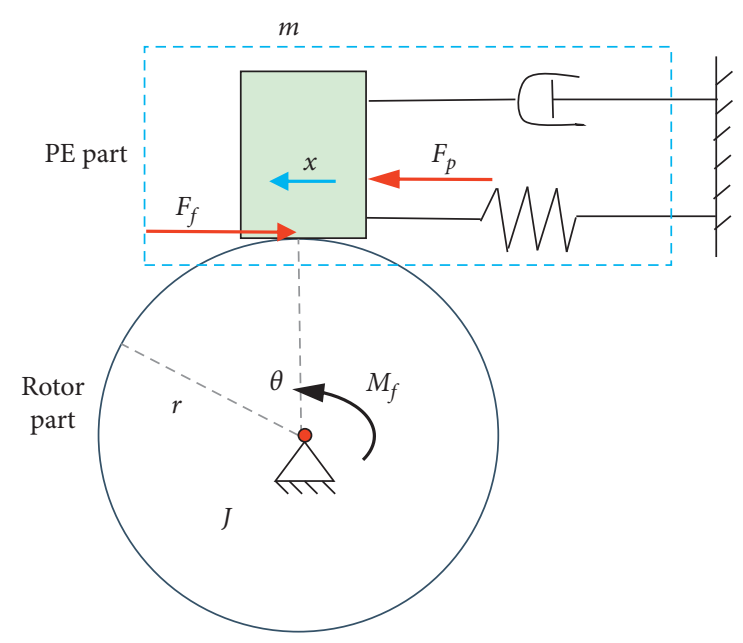

FIgURE 4: Dynamic model sketch.

compliance; $\sigma_{2}$ is the coefficient of viscous friction; $z$ is the average deflection of the bristles on the contact surface; $f_{s}$ is the stiction force; $f_{c}$ is the Coulomb friction force; and $v_{s}$ is the Stribeck velocity.

$$
\begin{gathered}
F_{f}=\sigma_{0} z+\sigma_{1} \frac{d z}{d t}+\sigma_{2} v, \\
\frac{d z}{d t}=v-\frac{|v|}{g(v)} z, \\
\sigma_{0} g(v)=f_{c}+\left(f_{s}-f_{c}\right) e^{-\left(v / v_{s}\right) 2},
\end{gathered}
$$

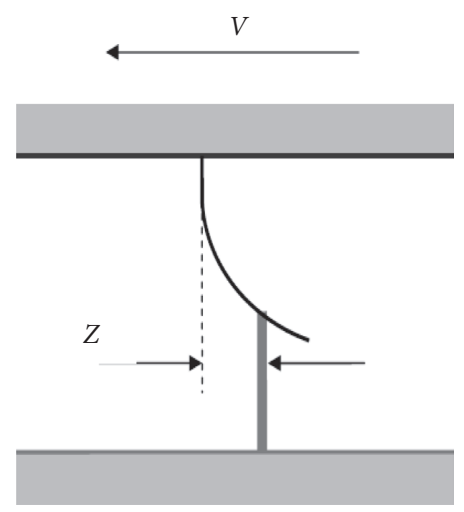

FIGURE 5: Deflected bristle in LuGre friction model.

3.2. Simulations. To investigate how the step displacement and speed of the actuator are affected by the parameters of the proposed dynamic model, such as $F_{p}$ and $F_{f}$, we built a Simulink model in MATLAB software and conducted simulations. The key parameters we used in the analysis are shown in Table 1.

Parameters $m, c$, and $k$ for a properly designed PE subsystem are easily measured in experiments. In the simulations, we keep the radius and rotational inertial of the rotor unchanged to evaluate the effect of the input waveform on the performance of the actuator.

Figure 6(a) presents the driving voltage signal of a $90 \mathrm{~V}$ sawtooth waveform applied to the PE subsystem. The failing edge of the voltage spans $40 \mu \mathrm{s}$, which is short enough to ensure a slip motion. Figure 6(b) depicts the displacement of the actuator under this driving voltage while Figure 6(c) presents the friction force. 
TABLE 1: Key parameters used in simulations.

\begin{tabular}{lc}
\hline Parameter & Value \\
\hline$m$ & $0.4 \mathrm{~g}$ \\
$c$ & $0.25 \mu \mathrm{F}$ \\
$k$ & $1 e-6 \mathrm{~N} / \mu \mathrm{m}$ \\
$k_{p} \cdot d_{e}$ & $0.75 \mathrm{~N} / \mathrm{V}$ \\
$r$ & $2.8 e-2 \mathrm{~m}$ \\
$J$ & $0.636 e-6$ \\
$M_{\text {load }}$ & $0 \mathrm{Nm}$ \\
$F c$ & $2 \mathrm{~N}$ \\
$F s$ & $2.4 \mathrm{~N}$ \\
$\sigma_{0}$ & $1 \mathrm{e} 6 \mathrm{~N} / \mathrm{m}$ \\
$\sigma_{1}$ & $8 \mathrm{Ns} / \mathrm{m}$ \\
$\sigma_{2}$ & $0 \mathrm{Ns} / \mathrm{m}$ \\
$v_{s}$ & $4 e-4 \mathrm{~m} / \mathrm{s}$ \\
\hline
\end{tabular}

Figure 6(b) shows evident stick-slip motion. In the stick phase, the voltage rises slowly, the rotor part and PE part move together, and the two drawn displacement lines overlap each other. In the slip phase, the voltage drops quickly, the PE part contracts quickly and returns to its original position while the rotor retracts slowly, and there is a sliding motion on the contacting surface and a step displacement is generated. After the stick phase, the rotor produced an angle displacement $\theta_{1}$, and then the rotor rotates backwards through displacement $\theta_{2}$ during the slip phase. The step angle displacement $\Delta \theta=\theta_{1}-\theta_{2}$.

Simple conclusions are drawn from the results shown in Figure 6. To achieve a higher resolution, a smaller step displacement is needed, and we hope to design a smaller $\theta_{1}$ and larger $\theta_{2}$ in a single cycle. In other words, the displacement of the PE part should be smaller. We can increase the mechanical stiffness of the PE part.

Other simulations are conducted to evaluate the actuator performance for different driving frequencies and amplitudes of the sawtooth wave.

We draw the following conclusions from the simulation results shown in Figures 7-9. When the properties of the PE part and rotor are decided, the performance of the piezoelectric actuator is dominated by the input driving voltage signal and the friction force in the stick-slip motion. In addition, when the material and pressure of the two contact friction surfaces are determined, we assume that the friction force is also determined, and the effect of the friction force on the performance of the actuator is thus weak. In this way, the frequency and amplitude of the driving voltage have the greatest effect on the performance of the actuator.

As the frequency of the driving voltage increases, the displacement $\theta_{1}$ in the stick phase and the displacement $\theta_{2}$ in the slip phase do not change. The step displacement therefore remains unchanged. However, in a same period, there are more steps contributing to the total displacement. When the amplitude of the driving voltage increases, the displacement $\theta_{1}$ in the stick phase increases while the displacement $\theta_{2}$ in the slip phase remains the same. The step angle displacement $\Delta \theta$ therefore increases.

The rotational speed of the actuator increases with the frequency of the driving voltage, while the angular step displacement increases with the amplitude of the driving voltage.

\section{Experiments and Actuator Characteristics}

4.1. Experiments. Figure 10 shows the prototype of the proposed piezoelectric rotary actuator. The actuator has dimensions of $56 \mathrm{~mm} \times 56 \mathrm{~mm} \times 12 \mathrm{~mm}$ and a mass of $51.68 \mathrm{~g}$. A series of experiments were conducted to test the performance and characteristics of the actuator.

Figure 11 shows the experimental system adopted to test the characteristics of the designed actuator. The waveform generator generated the required sawtooth waveform signal. The power amplifier amplified the signal from the waveform generator. The amplified signal was applied to the piezoelectric stacks in the actuator such that the actuator output rotary motion. A laser sensor with a resolution of $0.1 \mu \mathrm{m}$ was employed to measure the motion behavior of the actuator. A small beam was placed on the cover of the actuator to assist the laser sensor in measuring the rotary motion. When light hits the beam vertically, and the displacement measured by the laser sensor was about $100 \mu \mathrm{m}$ within a small angular displacement, the conversion formula for the output angular displacement is

$$
\theta(\mu r a d)=\frac{S(\mu m)}{R(m)},
$$

where $S$ is the displacement measured by the laser sensor and $R$ is the effective turning radius of the laser point on the beam. The personal computer was used to process the data from the laser sensor.

Figure 1(b) shows that the actuator contains two piezoelectric stacks. A series of experiments was conducted to investigate the performance of the prototype in different situations. Only one piezoelectric stack was installed and used in some experiments while two piezoelectric stacks were installed and used in other experiments.

4.2. Displacement Varying with Frequency. Figure 12 presents displacement data obtained directly from the laser sensor when the actuator installed with only one piezoelectric stack was driven by a sawtooth wave signal having a voltage of $93 \mathrm{~V}$ and symmetry of $100 \%$ and different frequencies.

First, we see that the displacement has a good linear relationship with time and the displacement has no visible backwards motion. This means that in the quick contraction stage, when the applied voltage decreases rapidly, the friction block slips on the bearing's outer ring, which remains still. The absence of backwards motion means that the actuator rotates stably and ideally.

Second, the velocity, which is presented as the slope of the line, increases with frequency. A higher frequency means that there are more steps in the same time period, resulting in larger displacement.

4.3. Velocity Varying with Frequency. To evaluate the performance of actuators at different frequencies of the driving 

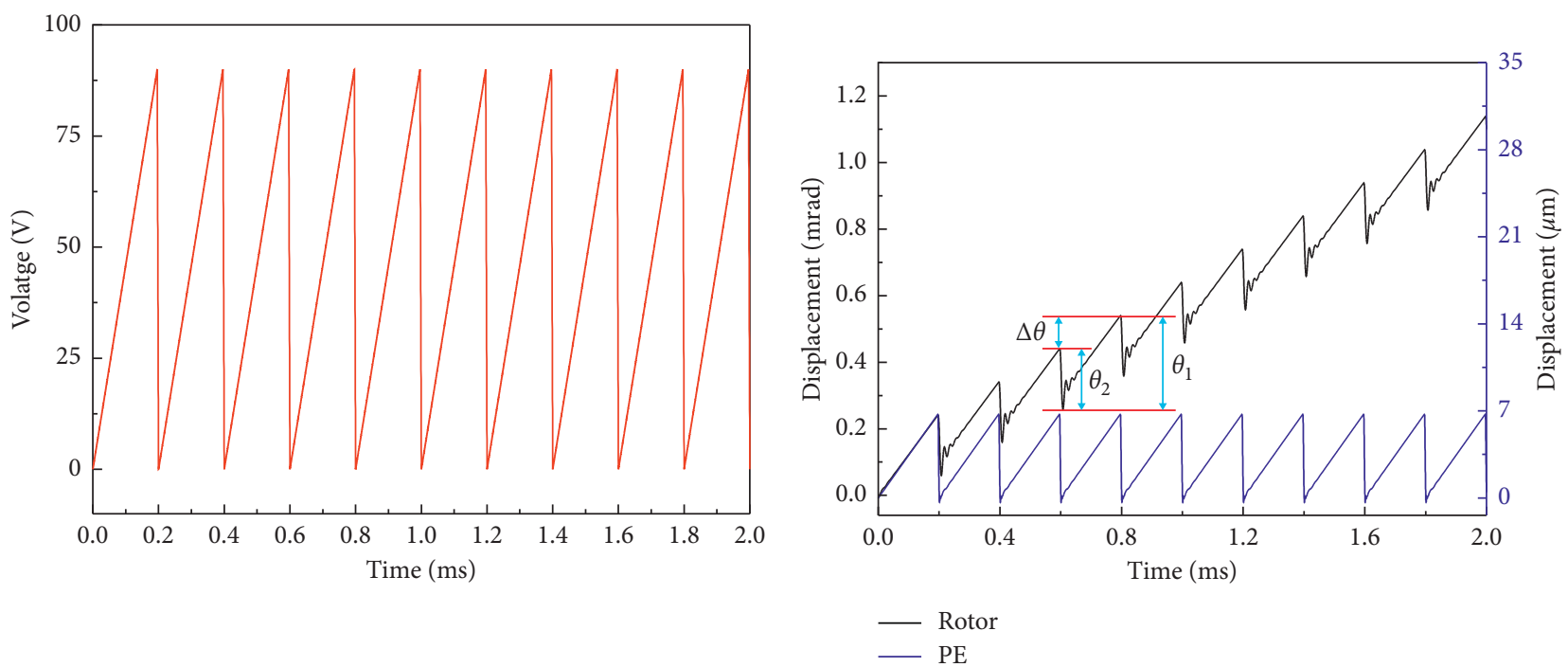

(a)

(b)

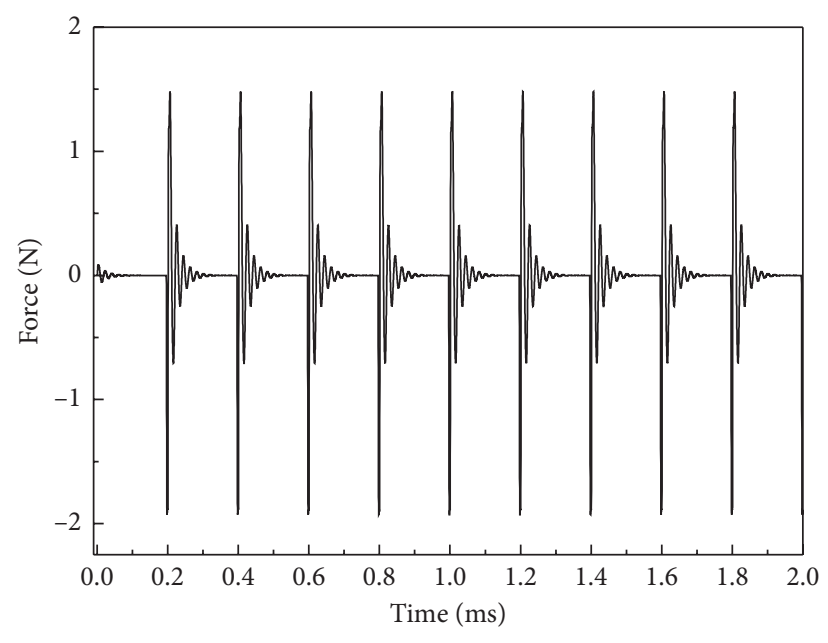

_ Friction force

(c)

FIGURE 6: Simulation results under sawtooth signal of $500 \mathrm{~Hz} 90$ V. (a) Driving voltage. (b) Displacement. (c) Friction force.

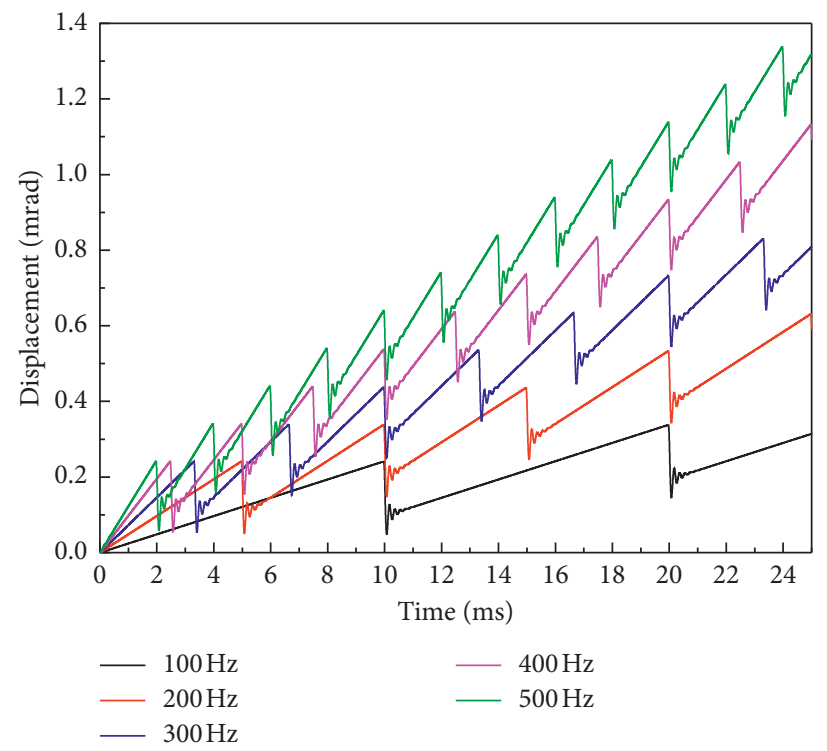

FIgURE 7: Simulation results of rotation displacement of the PA under $90 \mathrm{~V}$ sawtooth waveform. 


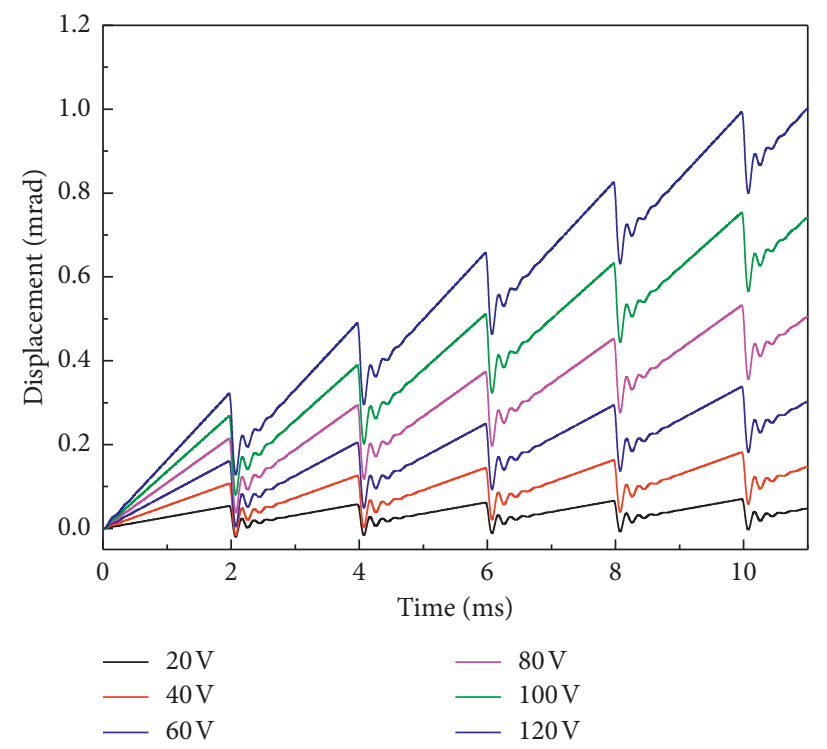

FIgURE 8: Simulation results of rotation displacement of the PA under $500 \mathrm{~Hz}$ sawtooth waveform at different amplitudes.

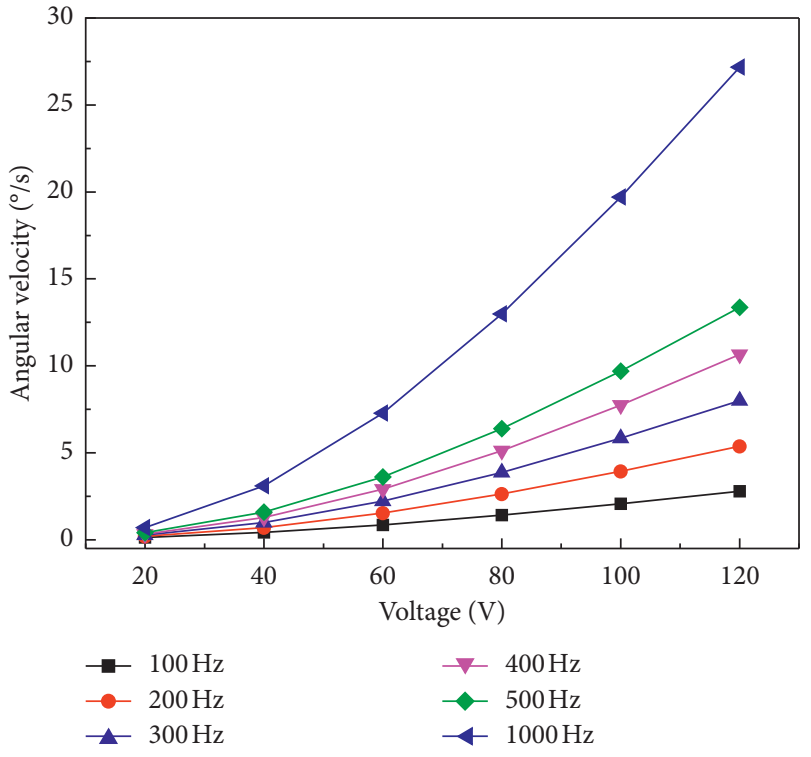

FIGURE 9: Simulation results of the PA's angular velocity under different frequencies and amplitudes of sawtooth waveform.

voltage, we applied a sawtooth wave signal having a voltage of $93 \mathrm{~V}$ and $100 \%$ or $0 \%$ symmetry on the piezoelectric stack. In this experiment, we installed one piezoelectric stack in the actuator. The sawtooth wave with $100 \%$ symmetry rotates the actuator clockwise while the sawtooth wave with $0 \%$ symmetry rotates the actuator anticlockwise.

The experimental results are shown in Figure 13. The clockwise motion and anticlockwise motion have nearly the same velocity at the same frequency, and both velocities increase with frequency. The relationship of the velocity and frequency has good linearity.

In the high-frequency range, the velocity does not increase with frequency. That is because of the bandwidth of

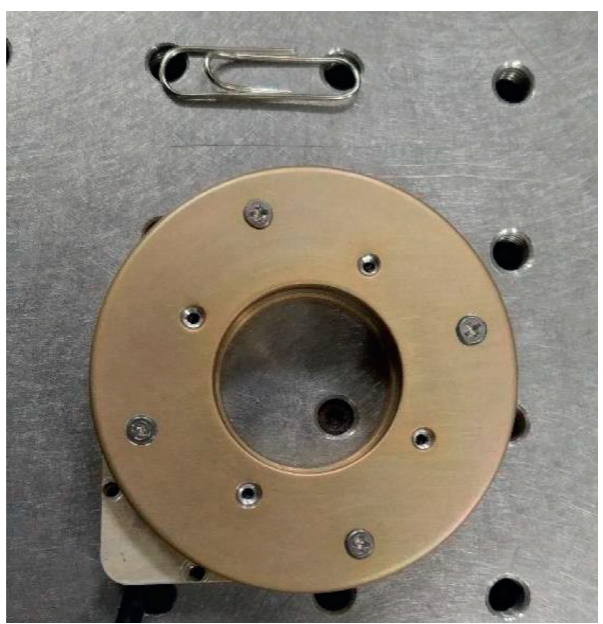

FIGURE 10: Prototype of the proposed piezoelectric rotary actuator.

the driving mechanism. When the frequency is increased beyond the bandwidth, the displacement output of the driving mechanism output would fall sharply, resulting in a decreased step angle. Besides, the time cost of the fast falling edge of the driving voltage is always fixed at about $100 \mu$ s due to the hardware limitation. An increase in the driving frequency reduces the total time of a single cycle waveform. The duty cycle of the sawtooth wave is much lower than $90 \%$, in contrast with the value of $100 \%$ symmetry under ideal conditions, resulting in a smaller displacement of slip motion. So the velocity in each direction thus remains nearly the same when the frequency is around $3000 \mathrm{~Hz}$.

4.4. Velocity Varying with Voltage. We applied a sawtooth wave signal having $100 \%$ symmetry and varying voltage and frequency to the piezoelectric stack, to clarify the 


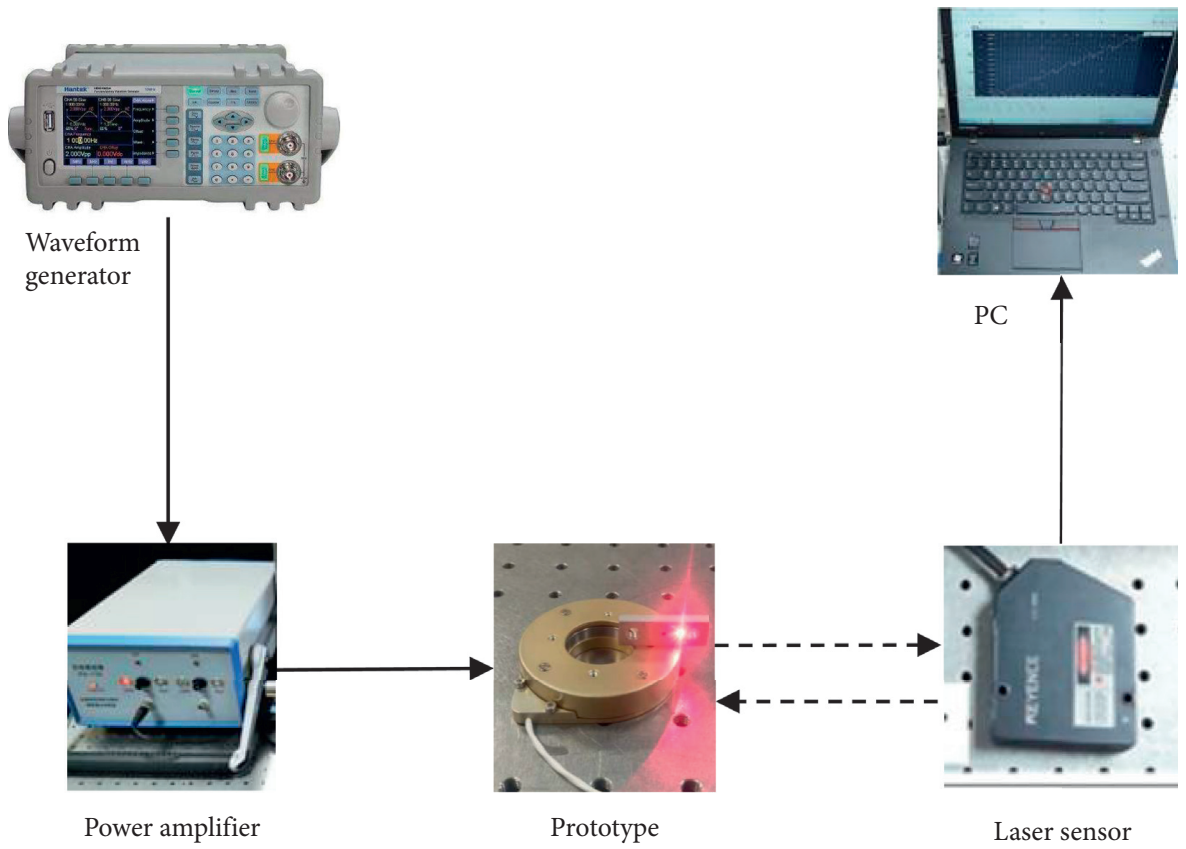

Figure 11: Experiment system.

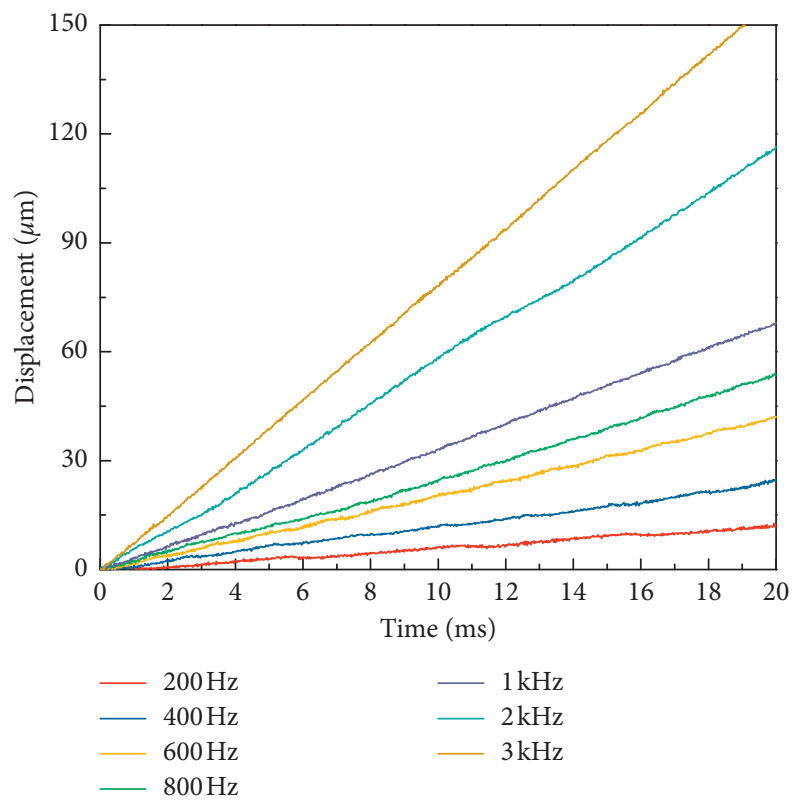

(a)

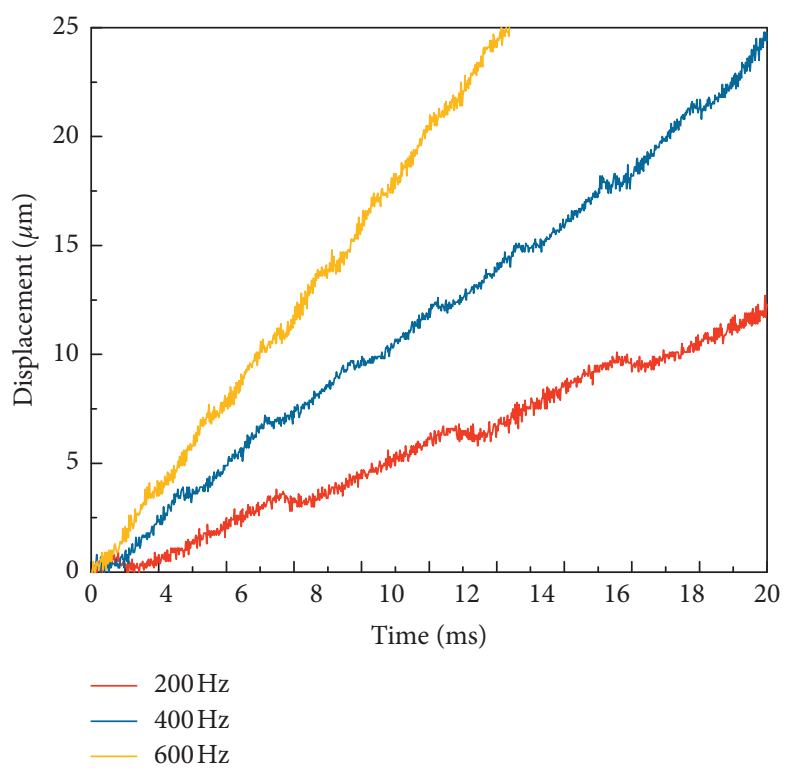

(b)

Figure 12: Output displacement under different frequencies of a $93 \mathrm{~V}$ sawtooth waveform.

relationship between the driving voltage and the actuator performance.

Figure 14 shows the relationship between the angular velocity and applied voltage. As the applied voltage increases, the angular velocity increases regardless of the frequency of the applied voltage. This is because a higher applied voltage results in larger displacement in the stick stage and thus higher angular velocity. The lowest voltage that produces stable rotation is approximately $13 V p-p$.

Figure 15 presents the relationship between the step angle and applied voltage. The step angle decreases as the applied voltage decreases or as the frequency of the applied voltage increases. A smaller step angle means that the actuator can reach higher resolution. The smallest step angle is 


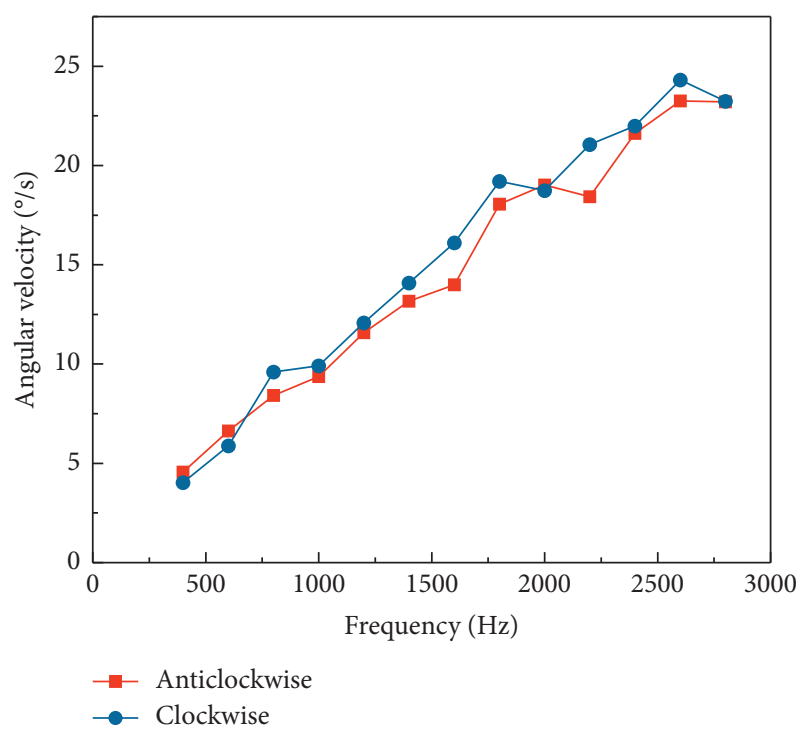

FIGURE 13: Velocity under different frequencies with a driving voltage of $93 \mathrm{~V}$.

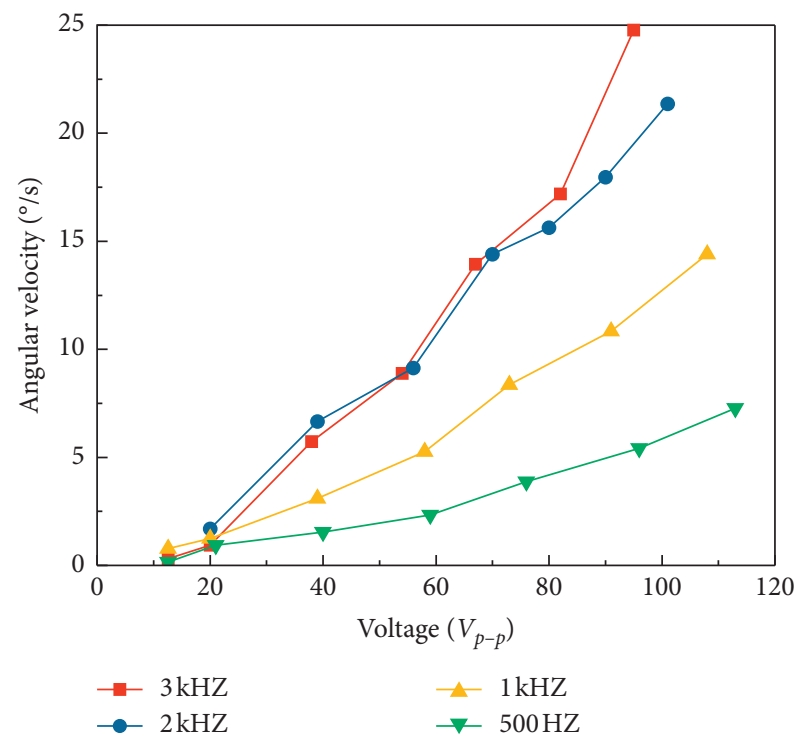

Figure 14: Velocity under different applied voltages.

approximately 0.2 millidegrees when then applied voltage is $13 \mathrm{Vp}-\mathrm{p}$ at a frequency of $3000 \mathrm{~Hz}$.

4.5. Velocity Varying with Number of Piezoelectric Stacks. The prototype of the proposed actuator worked well when only one piezoelectric stack was installed. The prototype also worked well when two piezoelectric stacks were installed and applied with the same sawtooth wave signal.

Figure 16 compares driving by one piezoelectric stack and driving by two piezoelectric stacks. When the frequency of the applied voltage is lower than $1600 \mathrm{~Hz}$, the angular velocities of the one piezoelectric actuator and two-piezoelectric actuator are similar in that both velocities increase

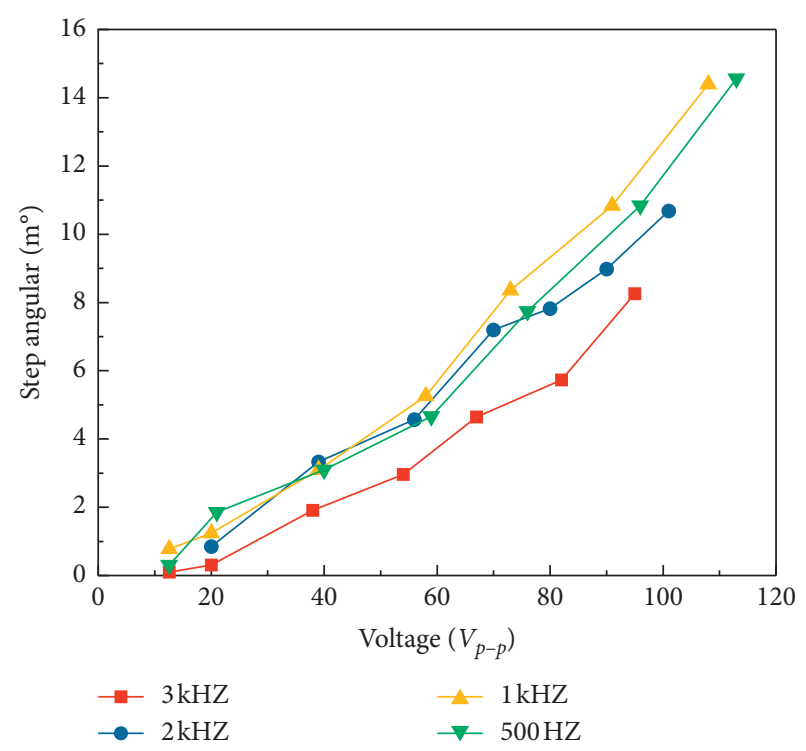

Figure 15: Step angular under different applied voltages.

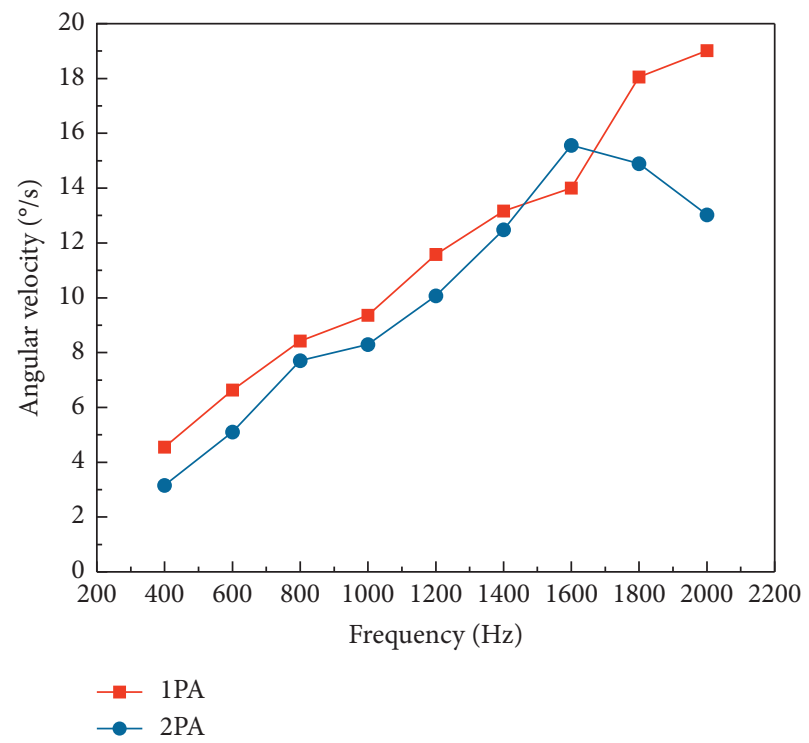

FIGURE 16: Driving by a sawtooth wave signal with $93 V p-p$ at different frequencies.

with frequency with good linearity. When the frequency is higher than $1600 \mathrm{~Hz}$, the velocity of the two-piezoelectric actuator decreases with increasing frequency. This is because the two piezoelectric stacks do not work perfectly at the same time and the two driving mechanisms have slightly different response times for the applied voltage. The difference is greater at a relatively high driving frequency. Another possible explanation is resonance.

Figure 17 shows the results obtained when we installed two driving mechanisms in the actuator but only applied voltage to one piezoelectric stack. This experiment simulates the situation that one piezoelectric stack is faulty and does not provide the expected driving motion. The red line shows 


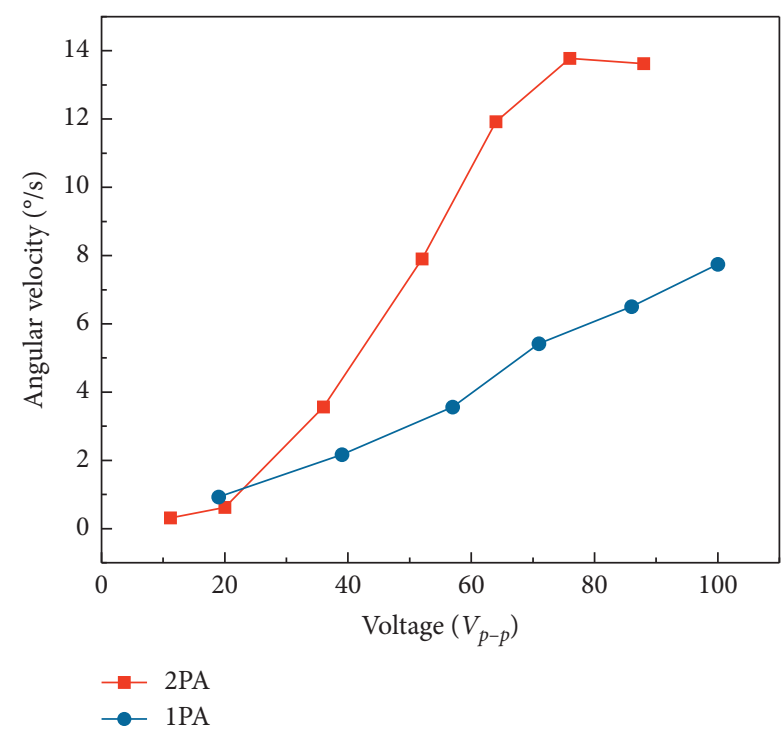

FIgURe 17: Driving by a sawtooth wave signal at $2000 \mathrm{~Hz}$ under different voltages.

results obtained when the voltage was applied to both piezoelectric stacks and the blue line shows results obtained when the driving voltage was applied only to one of the piezoelectric stacks.

It is seen that the actuator still works well under the extreme situation that only one piezoelectric stack does not work. The faulty piezoelectric stack does not provide a driving force but a friction force, working as an extra load for the actuator. The actuator rotates well at a lower speed. Irrespective of whether the driving voltage is applied to one or two piezoelectric stacks, the actuator rotates through $360^{\circ}$ under a low applied voltage. The angular velocity maintains a good linear relationship with the applied voltage at most frequencies.

\section{Conclusion}

In this work, following a comparison of the features of different types of piezoelectric actuator, we developed a novel stick-slip piezoelectric actuator for optical application. A dynamic model of the rotary piezoelectric actuator was established to simulate how the input driving voltage affects the stick-slip motion of the actuator. Simulation results show that the rotational speed of the actuator increases with the frequency of the driving voltage, while the angular step displacement increases with the amplitude of the driving voltage.

The actuator has been proposed, fabricated, and tested. An experimental system was built to evaluate the performance of the actuator at different frequencies, voltages, and numbers of driving piezoelectric stacks. Experimental results show that the minimal output stepping angle is $3.5 \mu \mathrm{rad}(0.2$ millidegrees) under a sawtooth waveform having a voltage of $13 \mathrm{~V}$ and frequency of $3000 \mathrm{~Hz}$ while the velocity reaches $0.44 \mathrm{rad} / \mathrm{s}\left(25^{\circ} / \mathrm{s}\right)$ under a sawtooth waveform having a voltage of $93 \mathrm{~V}$ and frequency of $3000 \mathrm{~Hz}$, while the stroke is infinite. The proposed actuator provides stable and accurate rotary motion and realizes a high velocity.

The actuator has merits of high resolution, good stability, high speed, infinite stroke, and compact size and has good application potential not only in an optical path adjustment system but also for other uses, such as positioning.

\section{Data Availability}

The data used to support the findings of this study are available from the corresponding author upon request.

\section{Conflicts of Interest}

The authors declare that they have no conflicts of interest.

\section{Acknowledgments}

This research was supported by the National Natural Science Foundation of China (Grant nos. 11902244 and 11872050).

\section{References}

[1] K. Uchino, Phase Transitions, vol. 88, pp. 342-355, Taylor \& Francis, Abingdon, UK, 2015.

[2] J. M. Breguet, W. Driesen, F. Kaegi, and T. Cimprich, "Organization of IEEE ICMA 2007 conference," in Proceedings of the 2007 IEEE International Conference on Mechatronics and Automation, pp. 57-62, Harbin, China, August 2007.

[3] Z. He, H. T. Loh, and E. H. Ong, "Reliability evaluation of piezoelectric micro-actuators with application in hard disk drives," IEEE Transactions on Magnetics, vol. 44, no. 11, pp. 3722-3725, 2008.

[4] J. Guo, S. K. Chee, T. Yano, and T. Higuchi, "Micro-vibration stage using piezo actuators," Sensors and Actuators A: Physical, vol. 194, pp. 119-127, 2013.

[5] T. Cheng, H. Li, M. He, H. Zhao, X. Lu, and H. Gao, "Investigation on driving characteristics of a piezoelectric stick-slip actuator based on resonant/off-resonant hybrid excitation," Smart Materials and Structures, vol. 26, no. 3, pp. 35-42, 2017.

[6] M. Sun, W. Huang, Y. Wang, Q. Lu, and Z. Su, "Research on a novel non-resonant piezoelectric linear motor with lever amplification mechanism," Sensors and Actuators A: Physical, vol. 261, pp. 302-310, 2017.

[7] Y. Tian, D. Zhang, and B. Shirinzadeh, "Dynamic modelling of a flexure-based mechanism for ultra-precision grinding operation," Precision Engineering, vol. 35, no. 4, pp. 554-565, 2011.

[8] G.-Y. Gu, L.-M. Zhu, and C.-Y. Su, "High-precision control of piezoelectric nanopositioning stages using hysteresis compensator and disturbance observer," Smart Materials and Structures, vol. 23, no. 10, p. 105007, 2014.

[9] H. C. Liaw and B. Shirinzadeh, "Constrained motion tracking control of piezoactuated flexure-based four-bar mechanisms for micro/nano manipulation," IEEE Transactions on Automation Science and Engineering, vol. 7, pp. 699-705, 2010.

[10] C.-H. Yun, T. Ishii, K. Nakamura, S. Ueha, and K. Akashi, “A high power ultrasonic linear motor using a longitudinal and bending hybrid bolt-clamped Langevin type transducer," Japanese Journal of Applied Physics, vol. 40, pp. 3773-3776, 2001. 
[11] Y. Liu, X. Yang, W. Chen, and D. Xu, "A bonded-type piezoelectric actuator using the first and second bending vibration modes," IEEE Transactions on Industrial Electronics, vol. 63, no. 3, pp. 1676-1683, 2016.

[12] S. Izuhara and T. Mashimo, "Design and evaluation of a micro linear ultrasonic motor," Sensors and Actuators A: Physical, vol. 278 , pp. 60-66, 2018.

[13] S.-T. Ho and S.-J. Jan, "A piezoelectric motor for precision positioning applications," Precision Engineering, vol. 43, pp. 285-293, 2016.

[14] P. E. Tenzer and R. B. Mrad, "A systematic procedure for the design of piezoelectric inchworm precision positioners," IEEE/ASME Transactions on Mechatronics, vol. 9, no. 2, pp. 427-435, 2004.

[15] H. Zhang, W. Huang, and L. Han, IOP Conference Series: Materials Science and Engineering, Vol. 381, Institute of Physics Publishing, Bristol, UK, 2018.

[16] S. Shao, S. Song, N. Chen, and M. Xu, "Structure and control strategy for a piezoelectric inchworm actuator equipped with MEMS ridges," Sensors and Actuators A: Physical, vol. 264, pp. 40-50, 2017.

[17] J. Li, H. Zhao, H. Qu et al., "A piezoelectric-driven rotary actuator by means of inchworm motion," Sensors and Actuators A: Physical, vol. 194, pp. 269-276, 2013.

[18] Z. M. Zhang, Q. An, J. W. Li, and W. J. Zhang, "Piezoelectric friction-inertia actuator-a critical review and future perspective," The International Journal of Advanced Manufacturing Technology, vol. 62, no. 5-8, pp. 669-685, 2012.

[19] M. Hunstig, "Piezoelectric inertia motors-a critical review of history, concepts, design, applications, and perspectives," Actuators, vol. 6, no. 1, p. 7, 2017

[20] D. Kang, M. G. Lee, and D. Gweon, "Development of compact high precision linear piezoelectric stepping positioner with nanometer accuracy and large travel range," Review of Scientific Instruments, vol. 78, no. 7, p. 075112, 2007.

[21] J. Y. Peng and X. B. Chen, "Modeling of piezoelectric-driven stick-slip actuators," IEEE/ASME Transactions on Mechatronics, vol. 16, no. 2, pp. 394-399, 2011.

[22] C. Edeler, I. Meyer, and S. Fatikow, "Modeling of stick-slip micro-drives," Journal of Micro-Nano Mechatronics, vol. 6, no. 3-4, pp. 65-87, 2011.

[23] M. Hunstig, T. Hemsel, and W. Sextro, "Stick-slip and slipslip operation of Piezoelectric inertia drives. Part I: ideal excitation," Sensors and Actuators A: Physical, vol. 200, pp. 90-100, 2013.

[24] S. Wang, W. Rong, L. Wang, Z. Pei, and L. Sun, "Design, analysis and experimental performance of a novel stick-slip type piezoelectric rotary actuator based on variable force couple driving," Smart Materials and Structures, vol. 26, 2017.

[25] T. Cheng, M. He, H. Li, X. Lu, H. Zhao, and H. Gao, "A novel trapezoid-type stick-slip piezoelectric linear actuator using right circular flexure hinge mechanism," IEEE Transactions on Industrial Electronics, vol. 64, no. 7, pp. 5545-5552, 2017.

[26] Q. S. Zhang, X. B. Chen, and Q. Yang, "Development and characterization of a novel piezoelectric-driven stick-slip actuator with anisotropic-friction surfaces," International Journal of Advanced Manufacturing Technology, vol. 61, no. 9-12, pp. 1029-1034, 2012.

[27] Y. Hu, R. Wang, J. Wen, and J.-Q. Liu, "A low-frequency structure-control-type inertial actuator using miniaturized bimorph piezoelectric vibrators," IEEE Transactions on Industrial Electronics, vol. 66, no. 8, pp. 6179-6188, 2019.
[28] G. Cheng, Y. Hu, J. Wen, P. Zeng, and C. Xing, "Piezoelectric inertial rotary actuators based on asymmetrically clamping structures," Sensors and Actuators A: Physical, vol. 223, pp. 125-133, 2015.

[29] Y. Zhang and T.-F. Lu, "On an equivalent model of multilayer piezoelectric actuators for facilitating finite element simulations," Microsystem Technologies, vol. 25, no. 12, pp. 4455-4464, 2019.

[30] C. C. de Wit, P. Lischinsky, K. J. Åström, and H. Olsson, “A new model for control of systems with friction," IEEE Transactions on Automatic Control, vol. 40, pp. 419-425, 1995. 